\title{
AN ANISOTROPIC CONSTITUTIVE RELATION FOR THE STRESS TENSOR OF A ROD-LIKE (FIBROUS-TYPE) GRANULAR MATERIAL
}

\author{
MEHRDAD MASSOUDI \\ Received 15 October 2004 \\ Dedicated to professor Stephan C. Cowin
}

We will derive a constitutive relationship for the stress tensor of an anisotropic rod-like assembly of granular particles where not only the transverse isotropy (denoted by a unit vector $\mathbf{n}$, also called the fiber direction) is included, but also the dependence of the stress tensor $\mathbf{T}$ on the density gradient, a measure of particle distribution, is studied. The granular media is assumed to behave as a continuum, and the effects of the interstitial fluid are ignored. No thermodynamical considerations are included, and using representation theorems, it is shown that in certain limiting cases, constitutive relations similar to those of the Leslie-Ericksen liquid crystal type can be obtained. It is also shown that in this granular model, one can observe the normal stress effects as well as the yield condition, if proper structures are imposed on the material coefficients.

\section{Introduction}

The primary approach for describing and analyzing coal furnaces and combustors has generally been accomplished through experimental studies where empirical correlations are used to describe the complex flows and chemical reactions that occur. In the last few decades, advanced combustion technologies have been developed with the intent of achieving higher overall system efficiencies and reduced environmental loading of air, water, and solid pollutants. Traditionally designers have relied on experiments to produce empirical formulas and correlations. One obvious difficulty with this approach is that, in general, changing the experiment or some of the conditions such as geometry, inlet conditions, particle loading, and so forth, may change the outcome and hence produce different correlations. The traditional approach is now being augmented with theoretical and computational modeling techniques, which provide the design engineers with the predictive capability and the freedom to choose and change conditions leading to a better design of combustors with higher efficiency, optimum geometry, less pollution, and so forth.

With additional need for fossil fuels, the amount of waste materials and the environmental issues dealing with their disposal also increase. One of the promising approaches 
is the development of coal/waste cofiring technology. For cofiring, biomass has been considered as one of the fuels. It is estimated that biomass constitutes $14 \%$ of the world energy use, which makes it the fourth largest energy source (Ekmann et al. [26]). Biomass can be considered any or a combination of wood residues, agricultural residues (crops, foods, animals), municipal solid waste, and so forth (Easterly and Burnham [24]). In addition to these, energy crops including short-rotation woody crops and herbaceous crops such as tall switchgrass, are thought to become the largest source of biomass in future. In general, biomass fuels are converted to energy via thermal, biological, and physical processes. Bridgwater [11] indicates that the three primary thermal processes for converting biomass to useful energy are combustion, gasification, and pyrolysis. Ekmann et al. [26] mention that from a technological point of view, for the biomass cofiring to become a viable source of energy “... both upstream and downstream impacts are important. Upstream impacts include handling, preparation (if any), and storage. Downstream ones include ash deposition (slagging and fouling), corrosion, and pollutants (reliable prediction of $\mathrm{NO}_{x}$ and $\mathrm{SO}_{x}$ reductions in particular)."

The major difficulties in modeling and using the cofiring of coal and biomass are (1) The biomass fuels, especially the switchgrass and wood-residue, are neither spherical nor disk-like in shape; most modeling approaches treat particles either as spherical or as disklike, with a shape factor to account for other shapes. (2) Since most of the biomass particles are slender and rod-like, the directionality or anisotropy associated with the axis of the body, that is, the orientation of the body, becomes an important controlling parameter. (3) For cofiring applications, the density of the biomass fuels is, in certain cases, significantly different from that of coal. These issues, in many ways, determine the efficiency of the mixing process. Most computational fluid dynamics (CFD) codes treat the particles as a homogenous continuous medium with correlations which depend on the diameter and density of these spherical particles.

In most fossil fuel combustion processes, there are several phases involved, whether the phases are of the same material or of different materials. It is therefore more accurate to refer to these studies as "multicomponent" problems; historically, two distinct approaches have been used to study these problems. In the first case, the amount of the dispersed (particulate or bubbly) phase is so small that the motion of this phase does not greatly affect or influence the motion of the continuous (or host) phase. This view is generally known as the "dilute phase approach," sometimes also called the Lagrangean approach, and is used extensively in applications such as atomization, sprays, and in flows where bubbles, droplets, and particles are treated as the dispersed phase (Sirignano [75], Crowe et al. [17], Sadhal et al. [72]). In the second approach, the two phases are interacting with each other to such an extent that each phase (or component) directly influences the motion and the behaviour of the other phase. This is known as the "dense phase approach," sometimes also called the Eulerian (or the two-fluid) approach. This method is used extensively in fluidization (Davidson et al. [18], Gidaspow [32]), gas-solid flows (Fan and Zhu [30]), pneumatic conveying (cf. Marcus et al. [48]), suspensions (Ungarish [88]), and is described for a variety of applications in general textbooks such as Soo [77], Rajagopal and Tao [63]. In most of the existing computer codes, the different phases are generally modeled as "fluids" and in certain cases the solid phase consisting of particles 


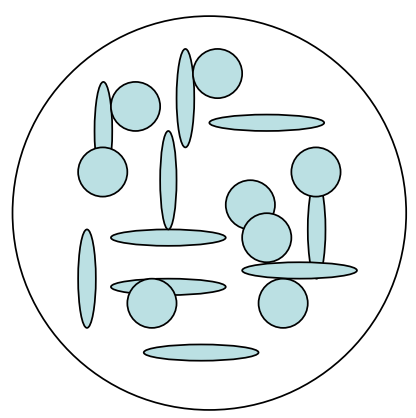

Figure 1.1. Mixture of coal-biomass gas.

of various shapes and sizes is modeled as granular materials, based either on continuum mechanics theories, or statistical theories such as the extension of the kinetic theory of gases or numerical simulation techniques. Therefore, in a combustor using the cofiring mechanism, where the primary fuel is coal and the secondary fuel is the biomass phase, we need to use a three-phase flow modeling approach (see Figure 1.1), with the gas (air) as the third phase. The coal and biomass particles have different chemical and thermomechanical properties. Since their densities, shapes, and sizes are so different, much of the biomass fuel is not properly mixed with the main fuel. In order to better understand the process of mixing and handling of these solid fuels, constitutive modeling of the stress tensors and the interaction mechanisms (see Massoudi $[49,50]$ ) are needed, especially in the fluid dynamics aspect of the process.

In recent years there has been a surge of interest in studies related to granular materials. Physicists, engineers, and mathematicians have begun to systematically look at the behaviour of particles in flowing and yielding conditions from different perspectives. These different approaches include experimental studies, statistical and continuum mechanics theories along with numerical simulations studies; with these, much light has been shed on the peculiar characteristics of powders. However, to this date there is not a single unified theory which can describe the response of granular materials to different flow conditions, concentrations, shapes and sizes, moisture content, and so forth. This, notwithstanding, is understandable, since granular materials behave similar to a fluid at times, and at other times similar to a solid. In addition to these, certain anisotropic characteristics, such as directionality of slender and thin fibrous-type granular materials and certain complex phenomena such as yield condition have made constitutive modeling truly a challenging task.

In an insightful essay, Behringer and Baxter (Mehta [55, page 107]) based on their experimental observations said, "In short, there is a need for a new kind of theory that includes both the unusual properties of dense granular flows and includes the given direction as a relevant variable." Two of the unusual properties of dense granular materials are (i) normal stress differences, and (ii) yield criterion. The first was observed by Reynolds and is normally called "dilatancy" $[69,70]$; this is a manifestation of nonequal normal stresses, similar to the rod-climbing and die-swell phenomena in rheology. The second 


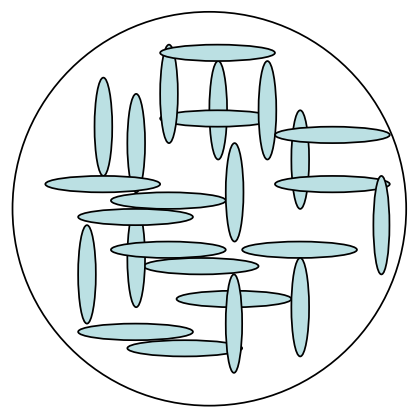

Figure 1.2. Biomass particles.

peculiarity is that for a granular solid to flow, there is often a yield stress below which the particles do not flow. This yield condition is often related to the angle of repose, friction, and cohesion, among other things. These two issues have made the constitutive modeling of granular materials very interesting. Among the most popular yield criteria is the Mohr-Coulomb criterion (Massoudi and Mehrabadi [52]), though by no means the only one. The model proposed and derived by them, based on the earlier work of Rajagopal and Massoudi [59] includes the effects of dilatancy and the Mohr-Coulomb yield condition. Earlier, Cowin $[15,16]$ had shown that by including the gradient of the bulk density as one of the important parameters in proposing a constitutive equation for the stress tensor, a theory for the flow of granular materials can be devised where not only a Mohr-Coulomb condition for limiting equilibrium is emerged in a natural way (because of the terms that could be identified with interparticle friction) but, additionally, the theory contains viscous terms corresponding to the "collisional" regime. One approach in the modeling of granular materials is to treat it as a continuum, which assumes that the material properties of the ensemble may be represented by continuous functions (Massoudi [51]). Another method is based on the techniques used in the kinetic theory of gases (Goldhirsch [33]). Another approach is computer or numerical modeling (Herrmann and Luding [39]). Recent comprehensive review articles by Savage [74], Hutter and Rajagopal [40], and de Gennes [19], and books by Nedderman [56], Mehta [55], Duran [23], and Antony et al. [5] address many of the interesting issues in the field of granular materials.

In this paper, we will derive a constitutive relationship for the stress tensor for an anisotropic rod-like assembly of granular particles (see Figure 1.2) where not only the transverse isotropy (denoted by a unit vector $\mathbf{n}$, also called the fiber direction) is included, but also the dependence of the stress tensor $\mathbf{T}$ on the density gradient, a measure of particle distribution, is considered. The granular media is assumed to behave as a continuum, and the effects of the interstitial fluid are ignored. No thermodynamical considerations are included, and using representation theorems, it is shown that in certain limiting cases, constitutive relations similar to those of the Leslie-Ericksen liquid crystal type can be obtained. It is also shown that in this granular model, one can observe the normal stress effects as well as the yield condition, if proper structures are imposed on the material coefficients. 
In Section 2, for the sake of brevity and conciseness, a brief review of constitutive modeling of the stress tensor $\mathbf{T}$ for three different classes of anisotropic materials, namely liquid crystals, fiber-reinforced composites, and granular materials are presented. In Section 3 , a general derivation for the constitutive relation is given for an anisotropic granular media where the effects of density gradients are included in the theory. In Section 4, three special cases of this general constitutive relation are discussed.

\section{A review of constitutive relations for anisotropic materials}

In this section, after discussing some general concepts in modeling anisotropic materials, we will focus on three specific classes of materials: (a) liquid crystals, (b) fiber-reinforced composites, and (c) granular materials. The objective is not to provide a comprehensive review of the subject, but rather to show certain similarities in the constitutive representation of the stress tensor.

The classical theories of continuum mechanics deal with the deformations and motions of materials that possess continuous mass densities. The general underlying assumption is the premise that any volume element, $\Delta v$, in a body can be taken to its limit, $d v$, without affecting the distribution of mass. According to this hypothesis, then, the identity of a material point in a volume element is lost, and its motion coincides with the motion of the center of mass of the body. For materials such as colloidal fluids, liquid crystals, granular, or composite materials, a theory that incorporates the micromotions of the particles contained in a material volume element, $\Delta v$, is needed. Materials possessing certain microstructures, for example, with the internal couples or couple stresses were first studied in the early twentieth century by D. Cosserat and F. Cosserat (Truesdell and Toupin [87]).

One of the basic challenges facing the researchers in the mathematical modeling of dense suspensions is the "slippery" procedure that is often required to go from the analytical and well-known classical results, usually valid for a single particle, or at the most for a few particles, to the not-so-well-known phenomena of interaction among particles and interaction between the particles and the host fluid. In the case of nonspherical particles, the classical study is that of Jeffery [42] who considered the motion of ellipsoidal particles in a viscous fluid. Generalizing this case to a suspension or an assembly of these particles is more difficult than generalizing the case of spherical particles, with the basic problem being that of the Stokes flow. The main reason for this difficulty is the orientation or the alignment of these nonspherical particles. To study this effect, there are at least two distinct yet related methods based on continuum mechanics. The first method is to use an orientation distribution function, whereby one derives orientation tensors to characterize the behaviour of these fibers. The idea of using orientation tensors to account, in an averaged sense, for the distribution of fibers in a fluid was suggested by Hand [37, 38]. The details of these techniques are given in Advani and Tucker [2], Advani [1], and Petrie [57]. The second method is to use the continuum mechanics theories whereby the microstructure is in some sense included in the theory, for example, as is done in the micropolar or director theories (Truesdell and Noll [85]). A very powerful use of this method is the theory of liquid crystals developed by Ericksen and later generalized by Hand, Leslie, and others. In this approach, a unit vector $\mathbf{n}$ is used as one of 
the independent constitutive variables, and as a result the stress tensor would depend on $\mathbf{n}$ and its derivatives, as well as other important constitutive parameters such as velocity, velocity gradient, temperature, and so forth, in an appropriate frame-invariant form.

In a seminal paper, Jeffrey [42] extends Einstein's results to the case of particles of ellipsoidal shape and showed that the particles increase the viscosity of the host fluid. Hand [37] later shows that the stress on the surface of a sphere referred to the axes coinciding with the principal axes of the ellipsoid, with some restrictions, is given by

$$
T_{i j}=-p_{0} \delta_{i j}+2 \mu D_{i j}+10 \mu\left(\frac{5 \phi}{R^{6}} \delta_{i j}+\frac{4 x_{i} x_{j} \phi}{R^{7}}-\frac{x_{i} \phi_{, j}}{R^{5}}-\frac{x_{j} \phi_{, i}}{R^{5}}\right)
$$

where

$$
\phi=A_{p q} x_{p} x_{q}
$$

where $D_{i j}$ is the symmetric part of the velocity gradient, $A_{p q}$ is a matrix whose components depend on the material properties and the values of $D_{i j}$ as $R \rightarrow \infty$, where $R$ is the radius of a sphere centered at the suspended particle, and $x_{i}=(x, y, z)$ are given by the ellipsoid of revolution

$$
\frac{x^{2}}{a^{2}}+\frac{y^{2}+z^{2}}{b^{2}}=1 .
$$

Hand [37] derives a theory for dilute suspensions of ellipsoidal particles and shows that if the flow is incompressible and laminar, by neglecting particle inertia, the stress tensor can be shown to be (Hand [37] shows that this equation is a special case of Ericksen's theory of anisotropic fluids)

$$
T_{i j}=-p_{0} \delta_{i j}+2 \mu D_{i j}+\frac{32 \pi \mu}{3 V} A_{i j}
$$

where

$$
V=\frac{4 \pi R^{3}}{3}
$$

Later, Hand [38] derives a more general theory for anisotropic fluids where he assumes that

$$
\mathbf{T}=\mathbf{T}(\mathbf{B}, \mathbf{D}),
$$

where $\mathbf{B}$ is a second-order tensor describing the microscopic structure of the fluid. A general expansion of this equation was given as

$$
\begin{aligned}
\mathbf{T}= & \beta_{0} \mathbf{1}+\beta_{1} \mathbf{B}+\beta_{2} \mathbf{D}+\beta_{3} \mathbf{B}^{2}+\beta_{4} \mathbf{D}^{2}+\beta_{5}(\mathbf{B D}+\mathbf{D B}) \\
& +\beta_{6}\left(\mathbf{B}^{2} \mathbf{D}+\mathbf{D B}^{2}\right)+\beta_{7}\left(\mathbf{B D}^{2}+\mathbf{D}^{2} \mathbf{B}\right)+\beta_{8}\left(\mathbf{B}^{2} \mathbf{D}^{2}+\mathbf{D}^{2} \mathbf{B}^{2}\right),
\end{aligned}
$$


where the $\beta$ 's are functions of the invariants of $\mathbf{B}$ and $\mathbf{D}$. By assuming that the fluid is incompressible and neglecting the $\mathbf{D}^{2}$ terms, Hand obtains a simplified form of $\mathbf{T}$ :

$$
\begin{aligned}
\mathbf{T}= & {\left[\sigma_{0}+\sigma_{1} \operatorname{tr}(\mathbf{B D})+\sigma_{2} \operatorname{tr}\left(\mathbf{B}^{2} \mathbf{D}\right)\right] \mathbf{1}+\left[\sigma_{3}+\sigma_{4} \operatorname{tr}(\mathbf{B D})+\sigma_{5} \operatorname{tr}\left(\mathbf{B}^{2} \mathbf{D}\right)\right] \mathbf{B} } \\
& +\sigma_{6} \mathbf{D}+\sigma_{7}(\mathbf{B D}+\mathbf{D B})+\sigma_{8}\left(\mathbf{B}^{2} \mathbf{D}+\mathbf{D B}^{2}\right),
\end{aligned}
$$

where the $\sigma$ 's are functions of the invariants of $\mathbf{B}$ only. He further assumes that

$$
\dot{B}_{i j}=F_{i j}\left(B_{k l}, \dot{x}_{p \cdot q}\right)
$$

and based on the results of Noll (Truesdell and Noll [85]) who had shown that the correct form of this equation in an invariant form under time-dependent orthogonal transformation is

$$
\hat{B}_{i j}=\dot{B}_{i j}-W_{i k} B_{k j}+B_{i k} W_{k j}
$$

where

$$
W_{i j}=\frac{1}{2}\left(\frac{\partial \dot{x}_{i}}{\partial x_{j}}-\frac{\partial \dot{x}_{j}}{\partial x_{i}}\right)
$$

Hand [38] then presents a general representation for (2.9) as

$$
\begin{aligned}
\dot{\mathbf{B}}= & \mathbf{W B}-\mathbf{B W}+\alpha_{0} \mathbf{1}+\alpha_{1} \mathbf{B}+\alpha_{2} \mathbf{D}+\alpha_{3} \mathbf{B}^{2}+\alpha_{4} \mathbf{D}^{2}+\alpha_{5}(\mathbf{B D}+\mathbf{D B}) \\
& +\alpha_{6}\left(\mathbf{B}^{2} \mathbf{D}+\mathbf{D B}^{2}\right)+\alpha_{7}\left(\mathbf{B D}^{2}+\mathbf{D}^{2} \mathbf{B}\right)+\alpha_{8}\left(\mathbf{B}^{2} \mathbf{D}^{2}+\mathbf{D}^{2} \mathbf{B}^{2}\right) .
\end{aligned}
$$

This constitutive relation along with that given by the stress tensor when substituted into the equations of conservation of mass and momentum provide ten equations to determine the ten unknowns $B_{i j}, u_{i}, p$. He shows that in a simple shear flow, this model of anisotropic fluid can predict normal stress differences.

2.1. Liquid crystals. Liquid crystals is the general name given to certain organic substances that have an independent thermodynamic state called a liquid crystalline state. For these substances, when the solid is melted, an anisotropic phase is produced which turns into an isotropic fluid at higher temperatures. The unusual phenomenon of interest here is that the physical properties of the fluid can be changed by various forces, such as electrical or magnetic fields, surface forces, and shear forces, that orient the molecules. In general, liquid crystals consist of large, relatively rigid molecules with one dimension larger than the others. For example, this can be visualized as a suspension of nonspherical particles. The boundaries of these particles are surfaces of revolutions and their preferred direction is the axis of revolution. Three types of liquid crystals are of significance: smectic, nematic, and cholesteric. (Leslie [46] defines these as "the smectic type is thought to have a stratified structure, the molecules lying in layers wit their long axes roughly normal to the planes of the layers. Their fluidity arises apparently though the layers slipping over each other. In the nematic and cholesteric liquid crystals, however, the long, rod-like molecules appear to be free to move randomly, except that they retain an orientation approximately parallel to that of their neighbors. The nematic and cholesteric seem to differ 
in that properties of the former are invariant with respect to certain reflections, whereas properties of the latter are not.")

Modern continuum theories of liquid crystals are due to Ericksen [27, 28, 29] who derived perhaps the simplest properly invariant theory of anisotropic fluids. He considered an incompressible fluid in which each particle has a single preferred direction, denoted by a unit vector $\mathbf{n}$ also called the director. His work was later generalized by Leslie $[44,45,46,47]$ and this formulation is known as Ericksen-Leslie theory of liquid crystals. In general $\mathbf{n}$ has its own motion, and the conservation equation for the flow of such a fluid are as follows.

(i) Mass:

$$
v_{i, i}=0 \quad \text { (for incompressible fluids). }
$$

(ii) Momentum:

$$
\rho \dot{v}_{i}=f_{i}+T_{j i, j}
$$

(iii) Angular momentum:

$$
\rho_{1} \ddot{n}_{i}=G_{i}+g_{i}+\Pi_{i j, j},
$$

where $\rho_{1}$ is a material constant (with the dimensions of moment of inertia per unit volume), $G_{i}$ the external director body force, $g_{i}$ the intrinsic body force, and $\Pi_{j i}$ the director surface stress.

The basic constitutive relations for the Ericksen-Leslie theory augment the above set of governing equations to provide a well-posed system. The constitutive relations are given (Chandrasekhar [14, page 97]):

$$
T_{j i}=T_{j i}^{0}+T_{j i}^{\prime}
$$

where

$$
\begin{gathered}
T_{j i}^{0}=-p \delta_{i j}-\frac{\partial F}{\partial n_{k, j}} n_{k, i}, \\
T_{j i}^{\prime}=\mu_{1} n_{k} n_{m} D_{k m} n_{i} n_{j}+\mu_{2} n_{j} N_{i}+\mu_{3} n_{i} N_{j}+\mu_{4} D_{j i}+\mu_{5} n_{j} n_{k} D_{k i}+\mu_{6} n_{i} n_{k} D_{k j},
\end{gathered}
$$

where $F$ is the free energy per unit volume:

$$
\begin{gathered}
F=\frac{1}{2}\left(k_{11}-k_{22}\right) n_{i, i} n_{j, j}+\frac{1}{2} k_{22} n_{i, j} n_{i, j}+\frac{1}{2}\left(k_{33}-k_{22}\right) n_{i} n_{j} n_{l, i} n_{l, j}, \\
N_{i}=\dot{n}_{i}-W_{i k} n_{k}, \quad D_{i j}=\frac{1}{2}\left(v_{i, j}+v_{j, i}\right), \quad W_{i j}=\frac{1}{2}\left(v_{i, j}-v_{j, i}\right),
\end{gathered}
$$

where $\mu_{1}, \ldots, \mu_{6}$ are the coefficients of viscosity (also known as Leslie coefficients), and $k_{11}, k_{22}, k_{33}$ are Frank's (Frank [31]) elastic constants.

Similarly,

$$
g_{i}=g_{i}^{0}+g_{i}^{\prime}
$$


where

$$
g_{i}^{0}=\gamma n_{i}-\beta_{j} n_{i, j}-\frac{\partial F}{\partial n_{i}}, \quad g_{i}^{\prime}=\lambda_{1} N_{i}+\lambda_{2} n_{j} D_{j i}
$$

where $\gamma, \beta_{j}$ (which arise due to the constraints of incompressibility and the director having fixed magnitude) are arbitrary constants, and

$$
\lambda_{1}=\mu_{2}-\mu_{3}, \quad \lambda_{2}=\mu_{5}-\mu_{6}
$$

And the director surface stress is given as

$$
\Pi_{j i}=\beta_{j} n_{i}+\frac{\partial F}{\partial n_{i, j}} .
$$

2.2. Fiber-reinforced materials. Sheet forming with continuous fiber-reinforced composites (McGuinness and Ó Brádaigh [54]) and fiber-reinforced thermoelastic materials (Johnson [43]) are but two of the most challenging problems in the manufacturing of composite materials. The rheological characteristics of a composite consisting of an isotropic matrix reinforced in one or two directions has been shown to behave as a highly anisotropic materials (Rogers [71]). Spencer [79] gave one of the earliest and most comprehensive (kinematic) theories for composite materials (Spencer [80] and Advani ([1] for more recent formulation and studies). Spencer [81] derives the basic set of equations for a composite material consisting of a matrix which is reinforced by two families of fibers, in two different directions defined by unit vectors $\mathbf{a}(\mathbf{x}, t)$, and $\mathbf{b}(\mathbf{x}, t)$, where the fibers are assumed to be continuously distributed. He furthermore makes the assumption that the fibers are convected with the material, that is,

$$
\frac{D a_{i}}{D t}=\frac{\partial a_{i}}{\partial t}+v_{j} \frac{\partial a_{i}}{\partial x_{j}}=\left(\delta_{i j}-a_{i} a_{j}\right) a_{k} \frac{\partial v_{j}}{\partial x_{k}} .
$$

A similar relationship also holds for $\mathbf{b}$. In most problems in classical fluid dynamics, the fluid is assumed to be incompressible, that is,

$$
\operatorname{tr} \mathbf{D}=D_{i i}=\frac{\partial v_{i}}{\partial x_{i}}=0 .
$$

If the condition of fiber inextensibility is also imposed, then

$$
a_{i} a_{j} D_{i j}=a_{i} a_{j} \frac{\partial v_{i}}{\partial x_{j}}=0, \quad b_{i} b_{j} D_{i j}=b_{i} b_{j} \frac{\partial v_{i}}{\partial x_{j}}=0 .
$$

Spencer [81] then shows that the stress for an anisotropic reinforced composite material which is incompressible and inextensible in the two fiber directions is given by

$$
\boldsymbol{\sigma}=-p \mathbf{1}+T_{a} \mathbf{a} \otimes \mathbf{a}+T_{b} \mathbf{b} \otimes \mathbf{b}+\boldsymbol{\tau},
$$

where $p$ is due to the incompressibility constraint, $T_{a}$ and $T_{b}$ are arbitrary tensions in the directions of $\mathbf{a}$ and $\mathbf{b}$, and $\otimes$ denotes the outer product. A constitutive relation for $\boldsymbol{\tau}$ is 
then required. He assumes that

$$
\tau=\tau(\mathbf{D}, \mathbf{a}, \mathbf{b})
$$

and shows that, using representation theorems, and considering (2.24) and (2.25), the most general form for $\boldsymbol{\tau}$ that is linear in $\mathbf{D}$ is

$$
\boldsymbol{\tau}=2 \eta \mathbf{D}+2 \eta_{1}(\mathbf{A D}+\mathbf{D A})+2 \eta_{2}(\mathbf{B D}+\mathbf{D B})+2 \eta_{3}\left(\mathbf{C D}+\mathbf{D C}^{\mathbf{T}}\right)+2 \eta_{4}\left(\mathbf{C}^{\mathrm{T}} \mathbf{D}+\mathbf{D C}\right),
$$

where $\eta$ 's are viscosities (which can be functions of $\mathbf{a} \cdot \mathbf{b}$ ), and

$$
\mathbf{A}=\mathbf{a} \otimes \mathbf{a}, \quad \mathbf{B}=\mathbf{b} \otimes \mathbf{b}, \quad \mathbf{C}=\mathbf{a} \otimes \mathbf{b}, \quad \mathbf{C}^{\mathrm{T}}=\mathbf{b} \otimes \mathbf{a},
$$

where the constraints (2.24) and (2.25) now become

$$
\operatorname{tr} \mathbf{D}=0, \quad \operatorname{tr} \mathbf{A D}=0, \quad \operatorname{tr} \mathbf{B D}=0 .
$$

An interesting and a special case of (2.27) is when there is material symmetry with respect to reflections in planes normal to the fiber direction, then (2.27) can be replaced by

$$
\tau=\tau(\mathrm{D}, \mathrm{A}, \mathrm{B})
$$

Spencer [83] derives constitutive relations for a much more general class of anisotropic fluids (with only one fiber direction a, although the same methodology can be extended to more than one fiber) which are also viscoelastic; specifically he generalizes the ReinerRivlin and the Rivlin-Ericksen second-order fluids. For example, the generalized secondorder transversely isotropic fluid has the following structure:

$$
\begin{aligned}
\boldsymbol{\sigma}= & -p \mathbf{1}+\nu_{o} \mathbf{a} \otimes \mathbf{a}+\nu_{T} \mathbf{A}_{\mathbf{1}}+\left(\nu_{L}-\nu_{T}\right)\left(\mathbf{a} \otimes \mathbf{a} \cdot \mathbf{A}_{\mathbf{1}}+\mathbf{A}_{\mathbf{1}} \cdot \mathbf{a} \otimes \mathbf{a}\right)+\eta_{1} \mathbf{A}_{\mathbf{2}} \\
& +\eta_{2} \mathbf{A}_{\mathbf{1}}{ }^{2}+\eta_{3}\left(\mathbf{a} \otimes \mathbf{a} \cdot \mathbf{A}_{\mathbf{2}}+\mathbf{A}_{\mathbf{2}} \cdot \mathbf{a} \otimes \mathbf{a}\right)+\eta_{4}\left(\mathbf{a} \otimes \mathbf{a} \cdot \mathbf{A}_{\mathbf{1}}{ }^{2}+\mathbf{A}_{\mathbf{1}}{ }^{2} \cdot \mathbf{a} \otimes \mathbf{a}\right),
\end{aligned}
$$

where

$$
\mathbf{A}_{1}=\mathbf{L}+\mathbf{L}^{T}, \quad \mathbf{A}_{2}=\frac{d \mathbf{A}_{1}}{d t}+\mathbf{A}_{1} \mathbf{L}+(\mathbf{L})^{T} \mathbf{A}_{1}, \quad \mathbf{L}=\operatorname{grad} \mathbf{u},
$$

and $\nu$ 's and $\eta$ 's are functions of the invariants

$$
\operatorname{tr} \mathbf{A}_{1}^{2}, \quad \mathbf{a} \cdot \mathbf{A}_{\mathbf{1}} \cdot \mathbf{a}, \quad \mathbf{a} \cdot \mathbf{A}_{\mathbf{1}}{ }^{2} \cdot \mathbf{a}, \quad \mathbf{a} \cdot \mathbf{A}_{\mathbf{2}} \cdot \mathbf{a} .
$$

Further restrictions can be obtained if the fibers are also inextensible (Spencer $[83,(40)]$ ).

It is known that the composite material is stiffer and stronger in the direction of "greatest orientation." In order to devise a rational way to describe fiber orientation, Advani and Tucker [2] advocate using the probability distribution function $\psi$ which is shown to depend on a unit vector $\mathbf{n}$ (along the fiber):

$$
\psi(\mathbf{n})=\frac{1}{4 \pi}+\frac{15}{8 \pi} b_{i j} f_{i j}(\mathbf{n})+\frac{315}{32 \pi} b_{i j k l} f_{i j k l}(\mathbf{n})+\cdots,
$$


where

$$
b_{i j}=a_{i j}-\frac{1}{3} \delta_{i j}, \quad f_{i j}(\mathbf{n})=n_{i} n_{j}-\frac{1}{3} \delta_{i j}, \quad a_{i j}=n_{i} n_{j},
$$

where a rate equation for $\mathbf{n}$ is given as (Advani and Tucker [2, (30)])

$$
\dot{n}_{i}=-\frac{1}{2}\left(\omega_{i j} n_{j}\right)+\frac{1}{2} \lambda\left(\dot{\gamma}_{i j} n_{j}-\dot{\gamma}_{k l} n_{k} n_{l} n_{i}\right)-D_{r} \frac{1}{\psi} \frac{\partial \psi}{\partial n_{i}},
$$

where $D_{r}$ is the rotary diffusivity, $\lambda$ is a parameter which is related to the shape of the particle, and

$$
\dot{\gamma}_{i j}=2 D_{i j}=\left(v_{i, j}+v_{j, i}\right), \quad \omega_{i j}=2 W_{i j}=\left(v_{i, j}-v_{j, i}\right) .
$$

It is noted that when $D_{r}=0$, the equation reduces to Jeffrey's equation for a single fiber. By using (2.37) and the conservation of mass, Advani and Tucker [3] obtain the following equation for $a_{i j}$ :

$$
\frac{D a_{i j}}{D t}=-\frac{1}{2}\left(\omega_{i k} a_{k j}-a_{i k} \omega_{k j}\right)+\frac{1}{2} \lambda\left(\dot{\gamma}_{i k} a_{i k}+a_{k j} \dot{\gamma}_{k j}-2 \dot{\gamma}_{k l} a_{j k l i}\right)+2 C_{I} \dot{\gamma}\left(\delta_{i j}-3 a_{i j}\right),
$$

where now another equation is needed for

$$
a_{i j k l}=n_{i} n_{j} n_{k} n_{l}
$$

To model the stress tensor, they note that for most suspensions of fibers in a Newtonian fluid, it is reasonable to assume

$$
T_{i j}=C_{i j k l} \dot{\gamma}_{k l}
$$

where

$$
\begin{aligned}
C_{i j k l}= & B_{1} a_{i j k l}+B_{2}\left(a_{i j} \delta_{k l}+a_{k l} \delta_{i j}\right)+B_{3}\left(a_{i k} \delta_{j l}+a_{i l} \delta_{j k}+a_{j l} \delta_{i k}+a_{j k} \delta_{i l}\right) \\
& +B_{4} \delta_{i j} \delta_{k l}+B_{5}\left(\delta_{i k} \delta_{j l}+\delta_{i l} \delta_{j k}\right)
\end{aligned}
$$

where the B's are material constants. Tucker and Advani (see [1, page 171]) show that the rate of change of the orientation matrix $a_{i j}$, in general, can be expressed as

$$
\frac{D a_{i j}}{D t}=\mathbf{f}\left(a_{k l}, D_{k l}\right) \text {. }
$$

They also give a general representation for predicting the viscosity of suspensions of fibers in a Newtonian fluid (see [1, equation (6.2.61), page 177]):

$$
T_{i j}=\eta_{s} D_{i j}+\eta_{s} \phi\left\{A D_{k l} a_{i j k l}+B\left[D_{i k} a_{k j}+a_{i k} D_{k j}\right]+C D_{i j}+2 F a_{i j} B_{r}\right\},
$$

where $D_{i j}$ is the rate of deformation tensor, $\eta_{s}$ is the solvent viscosity, $\phi$ is the particle volume fraction, $A, B, C$, and $F$ are material constants, and $B_{r}$ is the rotary diffusivity due to Brownian motion. 
2.3. Granular materials. Any theory attempting to describe the behavior of flowing granular materials should embody several features. For example, a bulk solid is not exactly a solid continuum since it takes the shape of the vessel containing it; it cannot be considered a liquid for it can be piled into heaps; and it is not a gas for it will not expand to fill the vessel containing it. The flow of granular materials strongly depends upon the distribution of the void space. From the observation/experimental point of view, the pioneering work of Bagnold [9] has led to many formulations of non-Newtonian models (Reiner [67], Astarita and Ocone [6]). Goodman and Cowin [34, 35] developed a continuum theory for representing the stresses that occur during the flow of granular materials. The pneumatic effects are neglected; that is, the theory assumes that the material contained in the voids is a gas that does not interact with the granules. The basic idea underlying their theory is that the concept of mass distribution must be extended to admit granular materials; that is, the mass distribution must be related to the volume distribution of granules. This is achieved by introducing an independent kinematical variable called the volume distribution function. They assumed that the material properties of the ensemble are continuous functions of position. This is equivalent to assuming that the material may be divided indefinitely without losing any of its defining properties. That is, a distributed volume,

$$
V_{t}=\int v d V
$$

and a distributed mass,

$$
M=\int \rho_{s} v d V
$$

can be defined, where the function $v$ is an independent kinematical variable called the volume distribution function and has the property

$$
0 \leq v(\mathbf{x}, t)<1
$$

The function $v$ is represented as a continuous function of position and time; in reality, $v$ in a granular system is either one or zero at any position and time, depending upon whether there is a granule or a void at that position. That is, the real volume distribution content has been averaged, in some sense, over the neighborhood of any given position. The classical mass density, $\rho_{s}$, is called the distribution mass density, or simply the distributed density. The classical mass density. The bulk density, $\rho$, is related to $\rho_{s}$ and $\nu$ through

$$
\rho=\rho_{s} \nu
$$

After postulating the existence of new concepts, such as the "balance of equilibrated force" or the "balance of equilibrated inertia," Goodman and Cowin $[34,35]$ proposed new balance relations in addition to the regular balance laws of continuum mechanics. Many of these ideas had already been proposed in other areas of mechanics, such as liquid crystals and micropolar materials. They also introduced a new form of the entropy inequality. 
They derived a constitutive equation for the Cauchy stress tensor based on the ideas of continuum mechanics, the restrictions imposed by the Clausius-Duhem inequality, the principle of frame-indifference, and incompressibility of the grains. They also assumed that the constitutive representations for the free energy, heat flux, dissipative parts of the stress, and intrinsic equilibrated body force depend linearly on temperature gradient, velocity gradients, and gradient of the volume distribution function. Thus, the equation defining a Coulomb granular material becomes

$$
\mathbf{T}=\left(\beta_{0}-\beta v^{2}+\alpha \nabla \nu \cdot \nabla \nu+2 \alpha \nu \Delta \nu\right) \mathbf{1}-2 \alpha \nabla \nu \otimes \nabla \nu+\lambda(\operatorname{tr} \mathbf{D}) \mathbf{1}+2 \mu \mathbf{D}
$$

or

$$
T_{i j}=\left(\beta_{0}-\beta v^{2}+\alpha \nu_{, k} \nu_{, k}+2 \alpha \nu \nu_{, k k}\right) \delta_{i j}-2 \alpha v_{, i} \nu_{, j}+\lambda D_{k k} \delta_{i j}+2 \mu D_{i j}
$$

where $\Delta$ is the Laplacian operator, $\otimes$ represents the outer (dyadic) product of two vectors. The coefficients $\beta_{0}, \beta$, and $\alpha$ are material constants; $\lambda$ and $\mu$ are, in general, functions of $\rho_{s}$ and $v$; and a comma denotes differentiation with respect to $\mathbf{x}$. Goodman and Cowin assumed that the stress tensor is obtained by the linear superposition of two parts: $\mathbf{T}^{0}$, a rate-independent (also referred to as equilibrium or nondissipative) part, which depends on the solids fraction $v$ and its gradients, and $\mathbf{T}^{*}$, a rate-dependent (viscous) part. Thus,

$$
\mathbf{T}=\mathbf{T}_{0}+\mathbf{T}_{*}
$$

Ehrentraut (Straughan et al. [84]) also points to the similarities between granular materials and anisotropic liquids. Experimental results of Villarruel et al. [89] point to many fascinating observations, for example, as they mention, "The most crucial difference between sphere and cylinder packings comes from the tendency of cylinders to align along their long axis, both with each other and with the container walls." Based on these and earlier observations, it is postulated here that the main reason for the poor mixing of coal-biomass is due to the fact that the "anisotropic" nature of the biomass rod-like particles is ignored. We therefore propose to derive a constitutive relation for this case. (In certain applications with a significant slip velocity between the particles and the host fluid, or when the velocity, temperature, and concentration of particles are of interest, one has to resort to multiphase theories. We will not consider this approach here, and refer the reader to the early works of Allen and Kline [4] who developed a modified form of the mixture theory with microstructure. Other works of interest are those of Sarkar and Lumley [73] and DeSilva [21, 22].)

\section{A constitutive relation for the stress tensor of (dense-phase) flowing rod-like granular materials}

We envision a body composed of voids and thin rod-like materials. The granules are long enough that they cannot be approximated as spherical or disk-like particles, and therefore a shape factor or an equivalent diameter cannot be used. The individual fiber has a principal direction, denoted with a unit normal vector $\mathbf{n}$. The bulk material is assumed to be dense enough that we can use continuum mechanics to formulate a stress tensor. 
As the bulk material is flowing, the individual fibers may have a tendency to distribute themselves, and therefore we think a measure of density variation should be included in our formulation. For the time being, we neglect the effects of the interstitial fluid, and therefore we will not use a multicomponent, that is, a mixture theory approach. Also, we assume that all fibers have the same temperature and therefore the effects of temperature are not included. We assume that the fibers are rigid, and the effects of moisture and electromagnetic fields are also ignored. The small scale forces such as Brownian diffusion, and so forth, are also ignored.

Let us assume that the stress tensor $\mathbf{T}$ can be expressed as

$$
\mathbf{T}=\mathbf{T}(\rho, \operatorname{grad} \rho, \mathbf{u}, \operatorname{grad} \mathbf{u}, \mathbf{n}) .
$$

Then frame-indifference (Truesdell and Noll [85]) implies

$$
\mathbf{T}=\mathbf{T}(\rho, \operatorname{grad} \rho, \mathbf{D}, \mathbf{n})
$$

where

$$
\mathbf{D}=\frac{1}{2}\left[\operatorname{grad} \mathbf{u}+(\operatorname{grad} \mathbf{u})^{T}\right]
$$

For simplicity, let us define

$$
\mathbf{m}=\operatorname{grad} \rho .
$$

Then we can write

$$
\mathbf{T}=\mathbf{T}(\rho, \mathbf{m}, \mathbf{n}, \mathbf{D}) .
$$

Let us define two second-order symmetric tensors associated with $\mathbf{m}$ and $\mathbf{n}$, as

$$
\begin{aligned}
\mathbf{M}=\mathbf{m} \otimes \mathbf{m} & =\operatorname{grad} \rho \otimes \operatorname{grad} \rho, \\
M_{i j}=\rho_{, i} \rho_{, j}, \quad \quad \mathbf{N} & =\mathbf{n} \otimes \mathbf{n}, \quad N_{i j}=n_{i} n_{j} .
\end{aligned}
$$

For an isotropic representation of $\mathbf{T}$, the generators for (3.5) are (Spencer [78], Zheng [93])

$$
\begin{gathered}
\mathbf{D}, \mathbf{D}^{2}, \mathbf{m} \otimes \mathbf{m}, \mathbf{n} \otimes \mathbf{n}, \\
\mathbf{m} \otimes \mathbf{D m}+\mathbf{D m} \otimes \mathbf{m}, \mathbf{m} \otimes \mathbf{D}^{2} \mathbf{m}+\mathbf{D}^{2} \mathbf{m} \otimes \mathbf{m}, \\
\mathbf{n} \otimes \mathbf{D n}+\mathbf{D n} \otimes \mathbf{n}, \mathbf{n} \otimes \mathbf{D}^{2} \mathbf{n}+\mathbf{D}^{2} \mathbf{n} \otimes \mathbf{n}, \\
\mathbf{m} \otimes \mathbf{n}+\mathbf{n} \otimes \mathbf{m}, \\
(\mathbf{m} \otimes \mathbf{D n}+\mathbf{D n} \otimes \mathbf{m})-(\mathbf{n} \otimes \mathbf{D} \mathbf{m}+\mathbf{D} \mathbf{m} \otimes \mathbf{n}) .
\end{gathered}
$$


The invariants associated with (3.7) are

$$
\begin{gathered}
\mathbf{D} \longrightarrow \operatorname{tr} \mathbf{D}, \operatorname{tr} \mathbf{D}^{2}, \operatorname{tr} \mathbf{D}^{3}, \\
\mathbf{m} \longrightarrow \mathbf{m} \cdot \mathbf{m}, \\
\mathbf{n} \longrightarrow \mathbf{n} \cdot \mathbf{n}, \\
\mathbf{m}, \mathbf{n} \longrightarrow \mathbf{m} \cdot \mathbf{n}, \\
\mathbf{m}, \mathbf{D} \longrightarrow \mathbf{m} \cdot \mathbf{D m}, \mathbf{m} \cdot \mathbf{D}^{2} \mathbf{m}, \\
\mathbf{n}, \mathbf{D} \longrightarrow \mathbf{n} \cdot \mathbf{D} \mathbf{n}, \mathbf{n} \cdot \mathbf{D}^{2} \mathbf{n}, \\
\mathbf{D}, \mathbf{m}, \mathbf{n} \longrightarrow \mathbf{m} \cdot \mathbf{D n}, \mathbf{m} \cdot \mathbf{D}^{2} \mathbf{n} .
\end{gathered}
$$

Using (3.7) and (3.8), the general representation for stress tensor given by (3.5) becomes (similar constitutive relations have been obtained, e.g., by Rajagopal and Wineman [65] and Rajagopal and Ruzicka [60] within the context of continuum mechanics of electrorhelogical materials)

$$
\begin{aligned}
\mathbf{T}= & a_{1} \mathbf{1}+a_{2} \mathbf{m} \otimes \mathbf{m}+a_{3} \mathbf{n} \otimes \mathbf{n}+a_{4}(\mathbf{m} \otimes \mathbf{n}+\mathbf{n} \otimes \mathbf{m})+a_{5} \mathbf{D}+a_{6} \mathbf{D}^{2} \\
& +a_{7}(\mathbf{m} \otimes \mathbf{D} \mathbf{m}+\mathbf{D} \mathbf{m} \otimes \mathbf{m})+a_{8}\left(\mathbf{m} \otimes \mathbf{D}^{2} \mathbf{m}+\mathbf{D}^{2} \mathbf{m} \otimes \mathbf{m}\right) \\
& +a_{9}(\mathbf{n} \otimes \mathbf{D} \mathbf{n}+\mathbf{D} \mathbf{n} \otimes \mathbf{n})+a_{10}\left(\mathbf{n} \otimes \mathbf{D}^{2} \mathbf{n}+\mathbf{D}^{2} \mathbf{n} \otimes \mathbf{n}\right) \\
& +a_{11}[(\mathbf{m} \otimes \mathbf{D n}+\mathbf{D n} \otimes \mathbf{m})-(\mathbf{n} \otimes \mathbf{D m}+\mathbf{D m} \otimes \mathbf{m})]
\end{aligned}
$$

or

$$
\begin{aligned}
T_{i j}= & a_{1} \delta_{i j}+a_{2} m_{i} m_{j}+a_{3} n_{i} n_{j}+a_{4}\left(m_{i} n_{j}+n_{i} m_{j}\right)+a_{5} D_{i j}+a_{6} D_{i j}^{2} \\
& +a_{7}\left(m_{i} D_{j k} m_{k}+D_{i k} m_{k} m_{j}\right)+a_{8}\left(m_{i} D_{j k}^{2} m_{k}+D_{i k}^{2} m_{k} m_{j}\right) \\
& +a_{9}\left(n_{i} D_{j k} n_{k}+D_{i k} n_{k} n_{j}\right)+a_{10}\left(n_{i} D_{j k}^{2} n_{k}+D_{i k}^{2} n_{k} n_{j}\right) \\
& +a_{11}\left[\left(m_{i} D_{j k} n_{k}+D_{i k} n_{k} m_{j}\right)-\left(n_{i} D_{j k} m_{k}+D_{i k} m_{k} n_{j}\right)\right],
\end{aligned}
$$

where $a_{1}-a_{11}$ are scalar functions of the set of invariants

$$
\begin{array}{lll}
I_{1}=\operatorname{tr} \mathbf{D}, & I_{2}=\operatorname{tr} \mathbf{D}^{2}, & I_{3}=\operatorname{tr} \mathbf{D}^{3}, \\
I_{4}=\operatorname{tr}[\mathbf{m} \otimes \mathbf{m}], & I_{5}=\operatorname{tr}[\mathbf{n} \otimes \mathbf{n}], & I_{6}=\operatorname{tr}[\mathbf{m} \otimes \mathbf{n}+\mathbf{n} \otimes \mathbf{m}], \\
I_{7}=\operatorname{tr}[\mathbf{m} \otimes \mathbf{D m}], & I_{8}=\operatorname{tr}\left[\mathbf{m} \otimes \mathbf{D}^{2} \mathbf{m}\right], & I_{9}=\operatorname{tr}[\mathbf{n} \otimes \mathbf{D n}], \\
I_{10}=\operatorname{tr}\left[\mathbf{n} \otimes \mathbf{D}^{2} \mathbf{n}\right], & I_{11}=\operatorname{tr}[\mathbf{m} \otimes \mathbf{D n}], & I_{12}=\operatorname{tr}\left[\mathbf{m} \otimes \mathbf{D}^{2} \mathbf{n}\right] .
\end{array}
$$

\section{Special cases}

Case 4.1. Let us assume

$$
a_{3}=a_{4}=a_{7}=a_{8}=a_{9}=a_{10}=a_{11}=0 .
$$

This case corresponds to a granular media, such as spherical particles, where there is 
a degree of symmetry and anisotropy does not play a role. However, density (or volume fraction) gradient is still important. For such a granular media, (3.8) becomes

$$
\mathbf{T}=b_{1} \mathbf{1}+b_{2} \mathbf{m} \otimes \mathbf{m}+b_{3} \mathbf{D}+b_{4} \mathbf{D}^{2},
$$

where $b_{1}-b_{4}$ are scalar functions of the appropriate invariants. Let us furthermore assume

$$
b_{1}=b_{1}(\rho, \operatorname{tr} \mathbf{D}, \operatorname{tr}(\mathbf{m} \otimes \mathbf{m})), \quad b_{2}=b_{2}(\rho), \quad b_{3}=b_{3}(\rho), \quad b_{4}=b_{4}(\rho) .
$$

Now, if we assume $b_{1}$ is given by

$$
b_{1}=\beta_{0}(\rho)+\beta_{1} \operatorname{grad} \rho \cdot \operatorname{grad} \rho+\beta_{2}(\rho) \operatorname{tr} \mathbf{D},
$$

then, (4.2) can be written as

$$
\mathbf{T}=\left[\beta_{0}(\rho)+\beta_{1}(\rho) \operatorname{grad} \rho \cdot \operatorname{grad} \rho+\beta_{2}(\rho) \operatorname{tr} \mathbf{D}\right] \mathbf{1}+b_{2} \operatorname{grad} \rho \otimes \operatorname{grad} \rho+b_{3} \mathbf{D}+b_{4} \mathbf{D}^{2}
$$

This equation was derived by Rajagopal and Massoudi [59]. A special case of this model, when $b_{4}=0$, has been used extensively by Massoudi and Rajagopal in a variety of applications (Massoudi et al. [53]).

For a simple shear flow, the velocity field $\mathbf{u}$ and the volume function $v$ are assumed to be of the form

$$
\mathbf{u}=u(y) \mathbf{i}, \quad v=v(y) .
$$

It then follows that

$$
\mathbf{D}=\frac{1}{2}\left(\begin{array}{ccc}
0 & u^{\prime} & 0 \\
u^{\prime} & 0 & 0 \\
0 & 0 & 0
\end{array}\right), \quad \mathbf{D}^{2}=\frac{1}{4}\left(\begin{array}{ccc}
\left(u^{\prime}\right)^{2} & 0 & 0 \\
0 & \left(u^{\prime}\right)^{2} & 0 \\
0 & 0 & 0
\end{array}\right)
$$

Also, notice that

$$
\begin{gathered}
\nabla v \cdot \nabla v=\left(\frac{d v}{d y}\right)^{2}, \quad \operatorname{tr} \mathbf{D}=0, \\
\nabla v \otimes \nabla v=\left(\frac{d v}{d y}\right)^{2} \mathbf{j} \otimes \mathbf{j} .
\end{gathered}
$$

Now, using (4.6)-(4.9) in (4.5), we find that

$$
\begin{gathered}
T_{x y}=\frac{1}{2}\left[\beta_{3}(\nu) \frac{d u}{d y}\right], \quad T_{x x}-T_{y y}=-\left[\beta_{4}(\nu)\right]\left(\frac{d \nu}{d y}\right)^{2}, \\
T_{y y}-T_{z z}=\left[\beta_{4}(\nu)\right]\left(\frac{d \nu}{d y}\right)^{2}+\left[\beta_{5}(\nu)\right]\left(\frac{d u}{d y}\right)^{2} .
\end{gathered}
$$


Therefore, we can see that the material exhibits both normal stress differences. If either the term $\beta_{5}(\nu) \mathbf{D}^{2}$ or $\beta_{4}(\nu) \nabla \nu \otimes \nabla \nu$ were absent from the constitutive expression in (4.5), the model would be capable of exhibiting only one of the normal stress differences. For example, in an idealized shear flow, it is possible to have constant solid volume fraction. In such a case the term corresponding to $\beta_{4}(\nu) \nabla \nu \otimes \nabla \nu$ vanishes and only one of the normal stress differences remains.

This equation can be decomposed in the following manner:

$$
\mathbf{T}=\mathbf{T}_{e}+\mathbf{T}_{d}
$$

where

$$
\begin{gathered}
\mathbf{T}_{e}=\left[\beta_{0}(\rho)+\beta_{1}(\rho) \operatorname{grad} \rho \cdot \operatorname{grad} \rho\right] \mathbf{1}+b_{2} \operatorname{grad} \rho \otimes \operatorname{grad} \rho, \\
\mathbf{T}_{d}=\left[\beta_{2}(\rho) \operatorname{tr} \mathbf{D}\right] \mathbf{1}+b_{3} \mathbf{D}+b_{4} \mathbf{D}^{2},
\end{gathered}
$$

where $\mathbf{T}_{e}$ and $\mathbf{T}_{d}$ can be thought of as the equilibrium (quasistatic) and dynamic parts of the stress tensor such that as $\mathbf{D} \rightarrow 0, \mathbf{T} \rightarrow \mathbf{T}_{e}$. This approach is used quite often in granular materials, and if we furthermore impose (Massoudi and Mehrabadi [52])

$$
\begin{gathered}
\beta_{0}=c \cot \phi, \\
\beta_{1}=\frac{\beta_{4}}{2}\left(\frac{1}{\sin \phi}-1\right),
\end{gathered}
$$

where $\phi$ is the internal angle of friction, $c$ is a coefficient measuring cohesion, and $\beta_{4}$ is related to $b_{2}$, then the yield condition, in the limiting equilibrium states, is the MohrCoulomb criterion. This indicates that

$$
|S|=b_{0} T+c,
$$

where $S$ and $T$ are the shear stress and normal stress, respectively, acting on a plane at a point; and $b_{0}$ is the coefficient of static friction related to the internal angle of friction $\phi$ through

$$
b_{0}=\tan \phi
$$

When cohesion is absent $(c=0)$, it is usual to call a granular medium an ideal one. One in which internal friction is absent $(\phi=0)$ is called an ideally cohesive medium.

Another interesting case is to see what happens to the equation of motion when $\mathbf{u}=\mathbf{0}$, which may correspond to a pile of particles stored in an infinite (long) container. In this case (4.5) reduces to

$$
\mathbf{T}_{e}=\left[\beta_{0}(\nu)+\beta_{1}(\nu) \operatorname{grad} \nu \cdot \operatorname{grad} \nu\right] 1+\beta_{4} \operatorname{grad} \nu \otimes \operatorname{grad} \nu
$$

Assuming that

$$
v=v(y)
$$


where $y$ is the positive upward direction, then the $y$-component of the equation of motion when $\mathbf{u}=\mathbf{0}$ becomes

$$
\frac{d}{d y}\left[\beta_{0}(\nu)+\beta_{1}(\nu)\left(\frac{d v}{d y}\right)^{2}\right]+\frac{d}{d y}\left[\beta_{4}(\nu)\left(\frac{d \nu}{d y}\right)^{2}\right]-\rho_{s} v g=0
$$

where $g$ is the acceleration due to gravity. Now if we further assume that

$$
\beta_{1}=\beta_{3}=0
$$

then (4.19) becomes

$$
\frac{d}{d y}\left[\beta_{0}(\nu)\right]=\rho_{s} \nu g
$$

Now if we assume a Taylor series expansion for $\beta_{0}$,

$$
\beta_{0}(\nu)=\beta_{01}+\beta_{0}^{\prime}(0) \nu+O\left|\nu^{2}\right|
$$

where $O\left|v^{2}\right|$ indicates terms of higher order than $\nu$. Now, if there are no particles, the stress tensor $\mathbf{T}$ should be zero. This indicates that (see Rajagopal and Massoudi [59])

$$
\beta_{01}=0
$$

and therefore,

$$
\beta_{0}(\nu)=\beta_{0}^{\prime}(0) \nu=k \nu
$$

where $k$ is a constant. Substituting (4.24) into (4.21) gives

$$
k \frac{d v}{d y}=\rho_{s} v g
$$

which can be integrated and its solution is given by

$$
\nu=A e^{\left(\rho_{s} g / k\right) y} .
$$

Evaluating this equation at two different heights $y_{1}$ and $y_{2}$, where $y_{2}>y_{1}$, we have

$$
\nu\left(y_{2}\right)=\nu\left(y_{1}\right) e^{\left(\rho_{s} g / k\right)\left(y_{2}-y_{1}\right)} .
$$


Physically we expect that under normal conditions, there would be more particles at the bottom of the container than at the top, and therefore,

$$
v\left(y_{1}\right) \succ v\left(y_{2}\right)
$$

which implies that

$$
\frac{\rho_{s} g}{k} \prec 0
$$

and since both $\rho_{s}$ and $g$ are positive, it follows that $k<0$.

Case 4.2. If in (4.5) we let

$$
\beta_{1}=\beta_{2}=b_{2}=0
$$

we will recover the constitutive relation for a Reiner-Rivlin-type fluid, if we also set

$$
\beta_{0}=-p
$$

Case 4.3. If in (3.9) we set

$$
a_{2}=a_{4}=a_{7}=a_{8}=a_{11}=0
$$

which means a flowing anisotropic material where density gradient does not have an impact on the stress, then we have

$$
\mathbf{T}=\alpha_{1} \mathbf{1}+\alpha_{3} \mathbf{n} \otimes \mathbf{n}+\alpha_{5} \mathbf{D}+\alpha_{6} \mathbf{D}^{2}+\alpha_{9}(\mathbf{n} \otimes \mathbf{D n}+\mathbf{D} \mathbf{n} \otimes \mathbf{n})+\alpha_{10}\left(\mathbf{n} \otimes \mathbf{D}^{2} \mathbf{n}+\mathbf{D}^{2} \mathbf{n} \otimes \mathbf{n}\right)
$$

or

$$
T_{i j}=\alpha_{1} \delta_{i j}+\alpha_{3} n_{i} n_{j}+\alpha_{5} D_{i j}+\alpha_{6} D_{i j}^{2}+\alpha_{9}\left(n_{i} D_{j k} n_{k}+D_{i k} n_{k} n_{j}\right)+\alpha_{10}\left(n_{i} D_{j k}^{2} n_{k}+D_{i k}^{2} n_{k} n_{j}\right)
$$

which is the same as Leslie-Ericksen equation (Leslie $[44,(1)]$ ), provided that

$$
\alpha_{1}=-p
$$

and the $\alpha$ 's are functions of

$$
n_{i} n_{i}, D_{i j} n_{i} n_{j}, D_{i k} D_{k j} n_{i} n_{j}, D_{i j} D_{i j}, D_{i k} D_{k j} D_{j i}
$$

Case 4.4. Let us define

$$
\mathbf{B}=\mathbf{n} \otimes \mathbf{n} .
$$


Let

$$
\begin{gathered}
a_{2}=a_{4}=a_{7}=a_{8}=a_{11}=0, \\
a_{1}=\sigma_{0}+\sigma_{1} \operatorname{tr}(\mathbf{B D})+\sigma_{2} \operatorname{tr}\left(\mathbf{B}^{2} \mathbf{D}\right), \\
a_{3}=\sigma_{3}+\sigma_{4} \operatorname{tr}(\mathbf{B D})+\sigma_{5} \operatorname{tr}\left(\mathbf{B}^{2} \mathbf{D}\right), \\
a_{6}=\sigma_{6}, \quad a_{9}=\sigma_{7}, \quad a_{10}=\sigma_{8} .
\end{gathered}
$$

Then, (3.9) becomes the equation proposed by Hand [38] (see (2.8) earlier).

Thus we can see that (3.9), in theory, includes a class of models developed for transversely isotropic fluids, fiber-reinforced fluids, and granular materials, if special structure and meaning are given to the various material coefficients.

\section{Summary and comments}

We have derived, using representation theorems, a frame-invariant anisotropic constitutive relation for the Cauchy stress tensor of a granular media consisting of rod-like particles (see (3.9)). This general equation not only depends on $\mathbf{D}$ (the symmetric part of the velocity gradient) and its higher-order powers, but also on the density gradient and the fiber direction. A few special cases of this model whereby certain coefficients are set to zero are also studied. It is shown that the normal stress effects can be observed in a simple shear flow. It is important to mention the basic assumptions and limitations of this derivation.

(1) Interstitial effects due to the presence of the fluid in the voids are completely ignored.

(2) Thermal effects are ignored and no thermodynamical issues (such as restrictions due to the second law) are considered.

(3) Although we have shown that under certain conditions, (3.9) can comply with the Mohr-Coulomb criterion (see (4.11)-(4.16)), other and more general yield conditions have been proposed, for example, by Rogers [71], and Spencer [82] for fiber-reinforced viscoplastic composites. These approaches are perhaps more suited for an anisotropic granular materials with a fiber direction $\mathbf{n}$ than the standard Mohr-Coulomb criterion.

(4) There are at least 11 material coefficients in (3.9) which in some ways have to be specified before a meaningful study can be done. Again, for certain cases, without performing any stability (Rajagopal et al. [64]) or thermodynamic analysis, we can gain some information about the sign of these parameters (see (4.17)-(4.29)). Some of the rheological properties can be measured, for example, using orthogonal rheometers (Rajagopal et al. [58]).

A general shortcoming of all higher-order or higher-gradient theories is the necessity of assigning boundary conditions for certain terms, which appear in the governing equations. Quite often these boundary conditions are not derived from first principles; instead they are given as ad hoc assumptions, or they are simply specified as mathematical conveniences. Sometimes experiments have been used successfully to specify these necessary additional boundary conditions. A second shortcoming of these higher theories, whether multipolar or director theories of liquid crystals, or turbulence theory, is the need for additional balance equations. Both of these shortcomings can be overcome 
through the application of multiple natural configurations theory developed by Rajagopal and coworkers. This theory has been successfully used for modeling non-Newtonian fluids (Rajagopal and Srinivasa [61]), anisotropic fluids (Rajagopal and Srinivasa [62]), to name only two applications.

\section{Acknowledgments}

I thank Professor K. R. Rajagopal for his comments and suggestions. I owe a great deal of gratitude to Professor S. C. Cowin whose research and writings in nonlinear continuum mechanics have influenced so much my professional/research life. His early work in mechanics of granular materials has laid the foundation of the continuum theories for such materials, and this paper is just an example and a simple extension of his ideas. This work is dedicated to Dr. Cowin for being such a source of inspiration for so many years for so many of us.

\section{References}

[1] S. G. Advani (ed.), Flow and Rheology in Polymer Composites Manufacturing, Elsevier, Amsterdam, 1994.

[2] S. G. Advani and C. L. Tucker III, The use of tensors to describe and predict fiber orientation in short fiber composites, J. Rheol. 31 (1987), no. 8, 751-784.

[3] Closure approximations for three-dimensional structure tensors, J. Rheol. 34 (1990), no. 3, 367-386.

[4] S. J. Allen and K. A. Kline, Theory of mixtures with microstructures, Z. Angew. Math. Phys. 20 (1969), 145-155.

[5] S. J. Antony, W. Hoyle, and Y. Ding, Granular Materials: Fundamentals and Applications, The Royal Society of Chemistry, Cambridge, 2004.

[6] T. Astarita and R. Ocone, Unsteady compressible flow of granular materials, Ind. Eng. Chem. Res. 38 (1999), no. 4, 1177-1182.

[7] R. J. Atkin and R. E. Craine, Continuum theories of mixtures: applications, J. Inst. Math. Appl. 17 (1976), no. 2, 153-207.

[8] Continuum theories of mixtures: basic theory and historical development, Quart. J. Mech. Appl. Math. 29 (1976), no. 2, 209-244.

[9] R. A. Bagnold, Experiments on a gravity free dispersion of large solid spheres in a newtonian fluid under shear, Proc. Roy. Soc. London Ser. A 225 (1954), no. 1160, 49-63.

[10] G. K. Batchelor, Sedimentation in a dilute dispersion of spheres, J. Fluid Mech. 52 (1972), 245268.

[11] A. V. Bridgwater, Renewable fuels and chemicals by thermal processing of biomass, Chem. Engrg. J. 91 (2003), no. 2-3, 87-102.

[12] A. V. Bridgwater, A. J. Toft, and J. G. Brammer, A techno-economic comparison of power production by biomass fast pyrolysis with gasification and combustion, Renewable Sust. Energy Rev. 6 (2002), no. 3, 181-248.

[13] P. Chadwick, Continuum Mechanics: Concise Theory and Problems, John Wiley \& Sons, New York, 1976.

[14] S. Chandrasekhar, Liquid Crystals, 2nd ed., Cambridge University Press, Cambridge, 1992.

[15] S. C. Cowin, A theory for the flow of granular materials, Powder Tech. 9 (1974), no. 2-3, 61-69.

[16] Constitutive relations that imply a generalized Mohr-Coulomb criterion, Acta Mech. 20 (1974), 41-46. 
[17] C. Crowe, M. Sommerfeld, and Y. Tsuji, Multiphase Flows with Droplets and Particles, CRC Press, Florida, 1998.

[18] J. F. Davidson, R. Clift, and D. Harrison (eds.), Fluidization, 2nd ed., Academic Press, Florida, 1985.

[19] P. G. de Gennes, Reflections on the mechanics of granular matter, Phys. A 261 (1998), no. 3-4, 267-293.

[20] P. G. de Gennes and J. Prost, The Physics of Liquid Crystals, 2nd ed., Oxford University Press, Oxford, 1993.

[21] C. N. DeSilva, A theory of concentrated suspensions, Acta Mech. 49 (1983), 221-239.

[22] _ Dilute suspensions, Acta Mech. 47 (1983), 1-14.

[23] J. Duran, Sands, Powders, and Grains, Springer, New York, 2000.

[24] J. L. Easterly and M. Burnham, Overview of biomass and waste fuel resources for power production, Biomass and Bioenergy 10 (1996), no. 2-3, 79-92.

[25] S. F. Edwards, The rheology of powders, Rheol. Acta 29 (1990), no. 6, 493-499.

[26] J. M. Ekmann, J. C. Winslow, S. M. Smouse, and M. Ramezan, International survey of cofiring coal with biomass and other wastes, Fuels Process. Tech. 54 (1998), 171-188.

[27] J. L. Ericksen, Anisotropic fluids, Arch. Rational Mech. Anal. 4 (1960), 231-237.

[28] - Theory of anisotropic fluids, Trans. Soc. Rheol. 4 (1960), 29-39.

[29] Equilibrium theory of liquid crystals, Advances in Liquid Crystals, vol. 2, Academic Press, New York, 1976, pp. 233-298.

[30] L.-S. Fan and C. Zhu, Principles of Gas-Solid Flows, Cambridge University Press, Cambridge, 1998.

[31] F. C. Frank, On the theory of liquid crystals, Disc. Faraday Soc. 25 (1958), 19-28.

[32] D. Gidaspow, Multiphase Flow and Fluidization: Continuum and Kinetic Theory Descriptions, Academic Press, Massachusetts, 1994.

[33] I. Goldhirsch, Rapid granular flows, Annu. Rev. Fluid Mech. 35 (2003), no. 35, 267-293.

[34] M. A. Goodman and S. C. Cowin, Two problems in the gravity flow of granular materials, J. Fluid Mech. 45 (1971), 321-339.

[35] - A continuum theory for granular materials, Arch. Rational Mech. Anal. 44 (1972), 249266.

[36] A. E. Green and R. S. Rivlin, The relation between director and multipolar theories in continuum mechanics, Z. Angew. Math. Phys. 18 (1967), 208-218.

[37] G. L. Hand, A theory of dilute suspensions, Arch. Rational Mech. Anal. 7 (1961), 81-86.

[38] A theory of anisotropic fluids, J. Fluid Mech. 13 (1962), 33-46.

[39] H. J. Herrmann and S. Luding, Modeling granular media on the computer, Contin. Mech. Thermodyn. 10 (1998), no. 4, 189-231.

[40] K. Hutter and K. R. Rajagopal, On flows of granular materials, Contin. Mech. Thermodyn. 6 (1994), no. 2, 81-139.

[41] H. M. Jaeger, S. R. Nagel, and R. P. Behringer, Granular solids, liquids, and gases, Rev. Modern Phys. 68 (1996), no. 4, 1259-1273.

[42] G. B. Jeffery, The motion of ellipsoidal particles immersed in a viscous fluid, Proc. Roy. Soc. London Ser. A 102 (1922), 161-179.

[43] A. F. Johnson, Rheological model for the forming of fabric-reinforced thermoplastic sheets, Composites Manufacturing 6 (1995), 153-160.

[44] F. M. Leslie, Some constitutive equations for anisotropic fluids, Quart. J. Mech. Appl. Math. 19 (1966), 357-370.

[45] - Some constitutive equations for liquid crystals, Arch. Rational Mech. Anal. 28 (1968), 265-283.

[46] Some thermal effects in cholesteric liquid crystals, Proc. Roy. Soc. London Ser. A 307 (1968), no. 1490, 359-372. 
[47] Theory of flow phenomena in liquid crystals, Adv. Liq. Crystals 4 (1979), 1-81.

[48] R. D. Marcus, L. S. Leung, G. E. Klinzing, and F. Rizk, Pneumatic Conveying of Solids, Chapman \& Hall, London, 1990.

[49] M. Massoudi, On the importance of material frame-indifference and lift forces in multiphase flows, Chem. Eng. Sci. 57 (2002), no. 17, 3687-3701.

[50] Constitutive relations for the interaction force in multicomponent particulate flows, Internat. J. Non-Linear Mech. 38 (2003), no. 3, 313-336.

[51] Constitutive modelling of flowing granular materials: a continuum approach, Granular Materials: Fundamentals and Applications (S. J. Antony, W. Hoyle, and Y. Ding, eds.), The Royal Society of Chemistry, Cambridge, 2004, pp. 63-107.

[52] M. Massoudi and M. M. Mehrabadi, A continuum model for granular materials: considering dilatancy, and the Mohr-Coulomb criterion, Acta Mech. 152 (2001), no. 1-4, 121-138.

[53] M. Massoudi, K. R. Rajagopal, and T. X. Phuoc, On the fully developed flow of a dense particulate mixture in a pipe, Powder Tech. 104 (1999), no. 3, 258-268.

[54] G. B. McGuinness and C. M. Ó Brádaigh, Development of rheological models for forming flows and picture-frame shear testing of fabric reinforced thermoplastic sheets, J. Non-Newtonian Fluid Mech. 73 (1997), no. 1-2, 1-28.

[55] A. J. Mehta (ed.), Granular Matter: An Interdisciplinary Approach, Springer, New York, 1994.

[56] R. M. Nedderman, Statics and Kinematics of Granular Materials, Cambridge University Press, Cambridge, 1992.

[57] C. J. S. Petrie, The rheology of fibre suspensions, J. Non-Newtonian Fluid Mech. 87 (1999), no. 2 3, 369-402.

[58] K. R. Rajagopal, G. Gupta, and R. C. Yalamanchili, A rheometer for measuring the properties of granular materials, Particulate Sci. Tech. 18 (2000), no. 1, 39-55.

[59] K. R. Rajagopal and M. Massoudi, A method for measuring material moduli for granular materials: flow in an orthogonal rheometer, Topical Report DOE/PETC/TR90/3, U. S. Department of Energy, Pennsylvania, 1990.

[60] K. R. Rajagopal and M. Ruzicka, Mathematical modeling of electrorheological materials, Contin. Mech. Thermodyn. 13 (2001), no. 1, 59-78.

[61] K. R. Rajagopal and A. R. Srinivasa, A thermodynamic frame work for rate type fluid models, J. Non-Newtonian Fluid Mech. 88 (2000), no. 3, 207-227.

[62]___ Modeling anisotropic fluids within the framework of bodies with multiple natural configurations, J. Non-Newtonian Fluid Mech. 99 (2001), no. 2-3, 109-124.

[63] K. R. Rajagopal and L. Tao, Mechanics of Mixtures, Series on Advances in Mathematics for Applied Sciences, vol. 35, World Scientific, New Jersey, 1995.

[64] K. R. Rajagopal, W. Troy, and M. Massoudi, Existence of solutions to the equations governing the flow of granular materials, European J. Mech. B Fluids 11 (1992), no. 3, 265-276.

[65] K. R. Rajagopal and A. S. Wineman, Flow of electrorheological materials, Acta Mech. 91 (1992), no. $1-2,57-75$.

[66] J. Rajchenbach, Granular flows, Adv. in Phys. 49 (2000), no. 2, 229-256.

[67] M. Reiner, A mathematical theory of dilatancy, Amer. J. Math. 67 (1945), 350-362.

[68] Rheology, Handbuch der Physik (S. Flugge, ed.), vol. 6, Springer, Berlin, 1958, pp. 434-550.

[69] O. Reynolds, On the dilatancy of media composed of rigid particles in contract with experimental illustrations, Phil. Mag. 20 (1885), no. S5, 469-481.

[70] - Experiments showing dilatancy, a property of granular material, possibly connected with gravitation, Proc. Royal. Inst. of Gr. Britain. 11 (1886), 354-363.

[71] T. G. Rogers, Rheological characterization of anisotropic materials, Composites 20 (1989), no. 1, $21-27$. 
[72] S. S. Sadhal, P. S. Ayyaswamy, and J. N. Chung, Transport Phenomena with Drops and Bubbles, Springer, New York, 1997.

[73] S. Sarkar and J. L. Lumley, A continuum two-fluid theory for dilute fiber suspensions, Acta Mech. 80 (1989), no. 3-4, 201-226.

[74] S. B. Savage, The mechanics of rapid granular flows, Adv. in Appl. Mech. 24 (1984), 289-366.

[75] W. A. Sirignano, Fluid Dynamics and Transport of Droplets and Sprays, Cambridge University Press, New York, 1999.

[76] G. F. Smith, On isotropic functions of symmetric tensors, skew-symmetric tensors and vectors, Internat. J. Engrg. Sci. 9 (1971), 899-916.

[77] S. L. Soo, Multiphase Fluid Dynamics, Science Press, Illinois, 1990.

[78] A. J. M. Spencer, Theory of invariants, Continuum Physics (A. C. Eringen, ed.), vol. 1, Academic Press, New York, 1971, pp. 239-353.

[79] Deformations of Fibre-Reinforced Materials, Oxford University Press, Oxford, 1972.

[80] A. J. M. Spencer (ed.), Continuum Theory of the Mechanics of Fibre-Reinforced Composites, CISM Courses and Lectures no. 282, Springer, New York, 1984.

[81] A. J. M. Spencer, Theory of fabric-reinforced viscous fluids, Composites: Part A 31 (2000), no. 12, $1311-1321$.

[82] _ A theory of viscoplasticity for fabric-reinforced composites, J. Mech. Phys. Solids 49 (2001), no. 11, 2667-2687.

[83] Some results in the theory of non-Newtonian transversely isotropic fluids, J. NonNewtonian Fluid Mech. 119 (2004), no. 1-3, 83-90.

[84] B. Straughan, R. Greve, H. Ehrentraut, and Y. Wang (eds.), Continuum Mechanics and Applications in Geophysics and the Environment, Springer, Berlin, 2001.

[85] C. Truesdell and W. Noll, The Nonlinear Field Theories of Mechanics, 2nd ed., Springer, Berlin, 1992.

[86] C. Truesdell and K. R. Rajagopal, An Introduction to the Mechanics of Fluids, Modeling and Simulation in Science, Engineering, and Technology, Birkhäuser Boston, Massachusetts, 2000.

[87] C. Truesdell and R. Toupin, The classical field theories, Handbuch der Physik, vol. III/1, Springer, Berlin, 1960, pp. 226-793.

[88] M. Ungarish, Hydrodynamics of Suspensions, Springer, New York, 1993.

[89] F. X. Villarruel, B. E. Lauderdale, D. M. Mueth, and H. M. Jaeger, Compaction of rods: relaxation and ordering in vibrated, anisotropic granular material, Phys. Rev. E 61 (2000), no. 6, 69146921.

[90] C. C. Wang, A new representation theorem for isotropic functions: An answer to Professor G. F. Smith's criticism of my papers on representations for isotropic functions. I. Scalar-valued isotropic functions, Arch. Rational Mech. Anal. 36 (1970), 166-197.

[91] A A new representation theorem for isotropic functions: An answer to Professor G. F. Smith's criticism of my papers on representations for isotropic functions. II. Vector-valued isotropic functions, symmetric ten tensor-valued isotropic functions, and skew-symmetric tensor-valued isotropic functions, Arch. Rational Mech. Anal. 36 (1970), 198-223.

[92] — Corrigendum to my recent papers on "Representations for isotropic functions", Arch. Rational Mech. Anal. 43 (1971), 392-395.

[93] Q.-S. Zheng, Theory of representations for tensor functions - A unified invariant approach to constitutive equations, Appl. Mech. Rev. 47 (1994), no. 11, 545-587.

Mehrdad Massoudi: National Energy Technology Laboratory, US Department of Energy, P.O. Box 10940, Pittsburgh, PA 15236, USA

E-mail address: massoudi@netl.doe.gov 


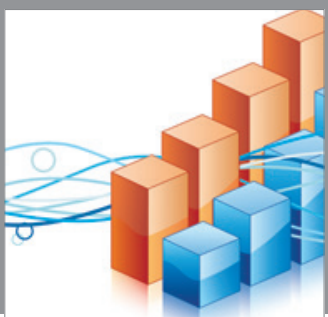

Advances in

Operations Research

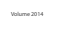

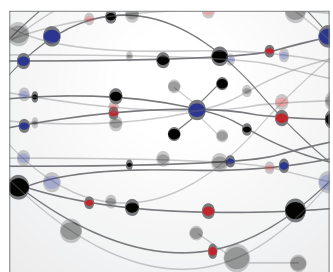

\section{The Scientific} World Journal
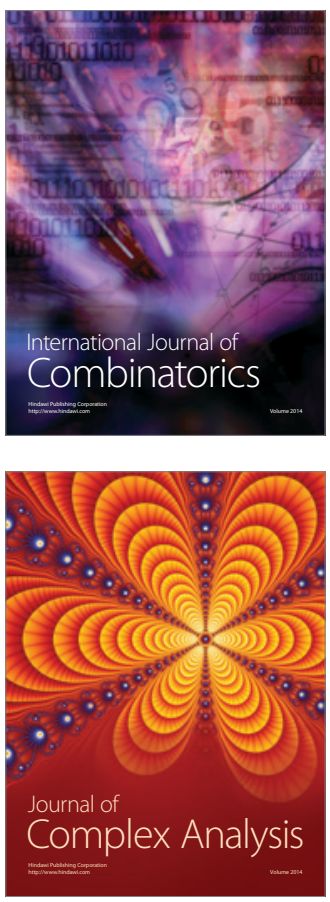

International Journal of

Mathematics and

Mathematical

Sciences
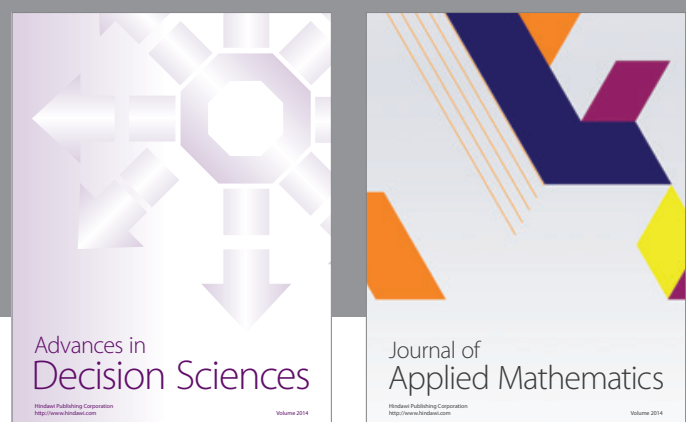

Journal of

Applied Mathematics
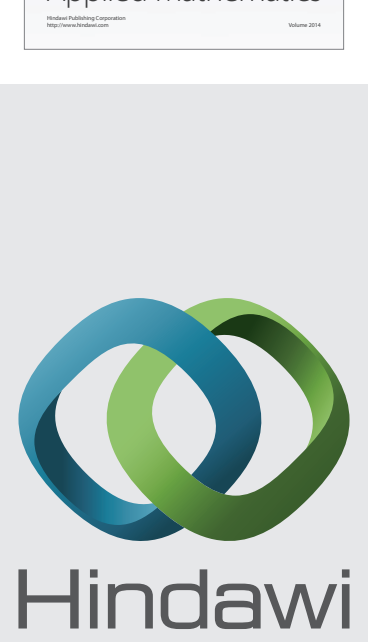

Submit your manuscripts at http://www.hindawi.com
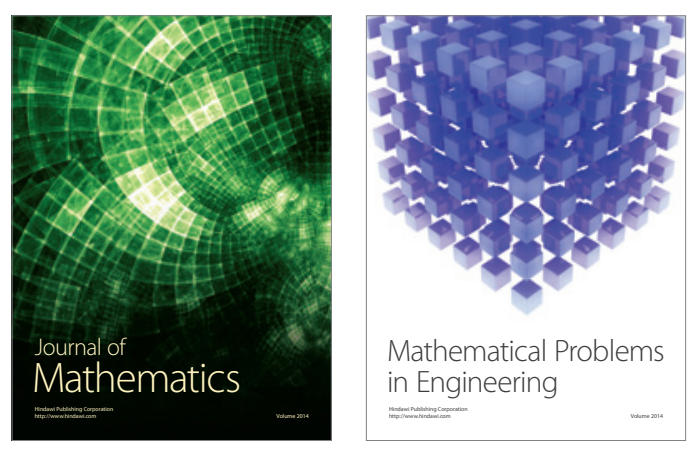

Mathematical Problems in Engineering
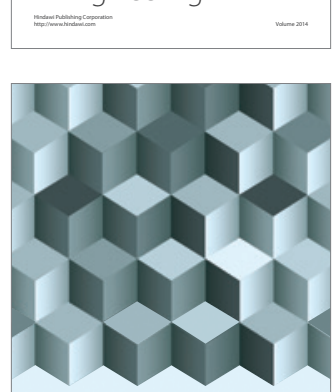

Journal of

Function Spaces
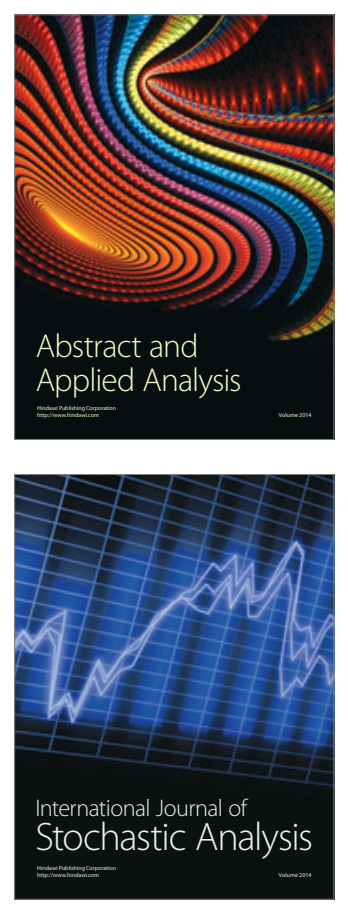

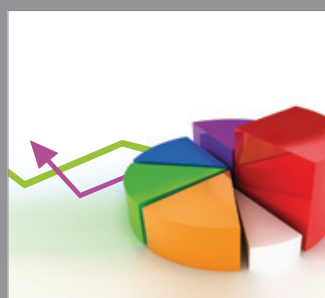

ournal of

Probability and Statistics

Promensencen
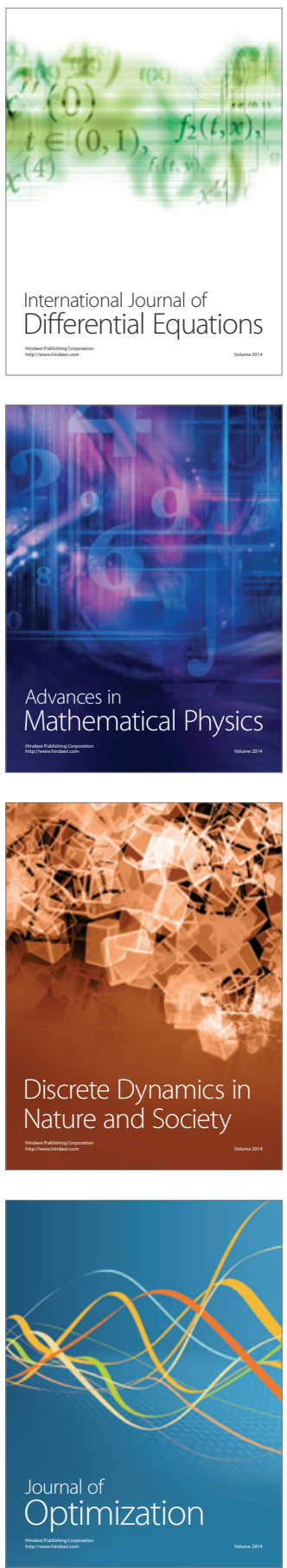